\title{
Efficacy and Tolerability of Solifenacin Fumarate with Overactive Bladder Patients: A Multicenter Observational Study
}

\author{
Jae Hun Shim, Se Young Choi, Joon Hee Gook, Yong-June Kim ${ }^{1}$, Woo Heon Cha², Dae Hee Kim ${ }^{3}$, Kyeong Hee Kim ${ }^{4}$, \\ Young Woong Park ${ }^{5}$, Jin Mo Um ${ }^{6}$, Il Sung Lim ${ }^{7}$, Kyung Keun Seo ${ }^{8}$, Kyu Seon Cho ${ }^{9}$, Young Jae Lee ${ }^{10}$, Mi-Kyung Lee ${ }^{11}$, \\ Tae-Hyoung Kim \\ Department of Urology, Chung-Ang University Hospital, Chung-Ang University College of Medicine, Seoul, ${ }^{1}$ Department of Urology, \\ Chungbuk National University College of Medicine, Cheongju, ${ }^{2}$ Gimcheon Medical Center, Gimcheon, ${ }^{3}$ Tower Urology Clinic, Seoul, ${ }^{4}$ Mizlove \\ Female Urology Clinic, Seoul, ${ }^{5}$ Naju Urology Clinic, Naju, ${ }^{6}$ Seoul Urology Group, Uijeongbu, ${ }^{7}$ Lim's Urology Clinic, Seoul, ${ }^{8}$ Urocenter \\ Urology Clinic, Cheonan, ${ }^{9}$ Seoul Top Urology Clinic, Seoul, ${ }^{10}$ Lee's Urologic Clinic, Incheon, ${ }^{11}$ Department of Laboratory Medicine, \\ Chung-Ang University, College of Medicine, Seoul, Korea
}

\begin{abstract}
Purpose: Overactive bladder $(\mathrm{OAB})$ is characterized by a series of highly prevalent symptoms among older adults. This study used the Overactive Bladder Symptom Score (OABSS) and Patient Perception of Bladder Condition (PPBC) tools to evaluate the efficacy and stability of solifenacin fumarate in the treatment of OAB. Materials and Methods: This was a prospective, multicenter, single-arm, 12-week study that enrolled $163 \mathrm{OAB}$ patients. The patients received $5 \mathrm{mg} /$ day of solifenacin fumarate. The changes in the OABSS, symptoms, and PPBC scores were evaluated at 0,4 , and 12 weeks. Subgroup analysis of the OABSS and PPBC scores based on sex, diabetes mellitus (DM) status, and body mass index (BMI) were also evaluated. Results: At the baseline (week 0), the mean OABSS for all patients was $8.45 \pm 2.38$ $(p=0.199)$. Subsequently, the mean OABSS declined to $5.41 \pm 2.69(p=0.255)$ at four weeks and $4.21 \pm 2.61(p=0.240)$ at 12 weeks. The OABSS subscore and PPBC score decreased significantly during the study $(p<0.01)$. After cases were stratified according to sex, DM status, and BMI, the mean OABSS (mean and subscore) and PPBC score at four and 12 weeks were also improved significantly relative to the baseline scores (both $\mathrm{p}<0.05$ ). The overall incidence of adverse events was $7.36 \%$ (12 cases), and three patients (1.82\%) permanently discontinued solifenacin fumarate because of the adverse events.

Conclusions: Solifenacin fumarate is a safe and effective treatment alternative for relieving OAB symptoms, considering the balance between the efficacy, patients' well-being, and tolerability.
\end{abstract}

Keywords: Urinary bladder, overactive; Urinary bladder; Solifenacin succinate

Copyright $\subset$ c) 2021, Korean Association of Urogenital Tract Infection and Inflammation. All rights reserved. (C) (1) (\$) This is an open access article distributed under the terms of the Creative Commons Attribution Non-Commercial License (http://creativecommons.org/licenses/by-nc/4.0) which permits unrestricted non-commercial use, distribution, and reproduction in any medium, provided the original work is properly cited.
Received: 18 November, 2020

Revised: 28 December, 2020

Accepted: 31 March, 2021
Correspondence to: Tae-Hyoung Kim
(i) https://orcid.org/0000-0002-0257-3449
Department of Urology, Chung-Ang University Hospital,
Chung-Ang University College of Medicine, 102
Heukseok-ro, Dongjak-gu, Seoul 06973, Korea
Tel: +82-2-3010-1818, Fax: +82-2-477-8928
E-mail: kthlmk@nate.com

\section{INTRODUCTION}

In 2002, the International Continence Society (ICS) defined irritable bladder as a group of symptoms, including urgency, with or without urge incontinence, with no urinary tract infection or other apparent causes, accompanied mainly by frequency and nocturia [1].

Few estimates of the prevalence of irritable bladder using 
the ICS definition have been published, but an irritable bladder can occur in all age groups. The incidence shows similar frequencies among male and female and tends to increase with age. A study of adults over 40 years old in Europe revealed $16.6 \%$ (males: $15.6 \%$, females: $16.4 \%$ ) to have experienced overactive bladder symptoms, and similar results were reported in the United States [2]. The Korea Urinary Incontinence Society investigated the prevalence of overactive bladder and lower urinary tract symptoms in 2000 male and female over 18 years of age using the 2002 ICS definition. In contrast to the above studies, the overall prevalence of irritable bladder was $12.2 \%$, with similar rates among male and female (male: 10.0\%, female: 14.3\%) [3]. This was similar to the prevalence observed in three European countries and Canada in 2006 (total: 11.8\%, male: 10.8, female: 12.8\%) [4], where the prevalence also increased with age. The overall prevalence in those over 40 years old was $14.9 \%$ (male: $11.2 \%$, female: $18.4 \%$ ) [3]. Based on this, and considering the adult population in Korea, 6 million people are estimated to have overactive bladder symptom.

Patients with irritable bladder symptoms tend to be isolated from social activity and interpersonal relationships. Moreover, the effect of irritable bladder on the quality of life is greater than that of diabetes mellitus (DM). Several studies have reported a high risk of depression associated with overactive bladder [5,6]. Furthermore, the risk of falling among individuals with urge incontinence is approximately $30 \%$ higher than those without urge incontinence and the risk of fracture is $3 \%$ higher [7].

The Overactive Bladder Symptom Score (OABSS) is used in the diagnosis of OAB [8]. OABSS is the most widely used questionnaire that is very useful for diagnosis and evaluating the subsequent treatment responses. The questionnaire consists of four questions (frequency [Q1], nocturia [Q2], urgency [Q3], incontinence [Q4]) that assess the symptoms from the patient's perspective. The diagnosis of $O A B$ is defined as OABSS urgency sub-score has more than 2 points and the total score is three or more. Severity of symptom was categorized as mild (OABSS: 55 ), moderate (OABSS: 611 ), and severe (OABSS: $\geq 12$ ). Total scores of $\leq 5,6-11$, and $\geq 12$ are considered mild, less severe, and severe, respectively [9]. The Patient Perception of Bladder Condition (PPBC) score is that the patient's bladder condition is divided into six items, from no problems to a severe problem.

Antimuscarinic agents are the primary treatment option for overactive bladder. This is because the mechanism underlying the development of urgency is sensory hyperreactivity of the bladder and detrusor muscle; acetylcholine is involved in both mechanisms. The detrusor muscle and sensory nerve receptors for acetylcholine are the muscarinic receptors. Local detrusor muscle contraction is caused by acetylcholine acting on the M3 muscarinic receptors, which antimuscarinic agents can effectively block. On the other hand, as acetylcholine secretion is upregulated during urination, normal urination is possible because a competitive antagonist, an antimuscarinic agent, does not block detrusor muscle contraction. Antimuscarinic drugs increase the bladder volume and ameliorate the urgency, overactive bladder, frequency, and nocturia associated with overactive bladder [10].

Solifenacin fumarate is an antimuscarinic agent that effectively blocks the M1 and M3 muscarinic receptors and has high bladder selectivity, making it useful for improving the symptoms of an irritable bladder [11]. The adverse effects of solifenacin fumarate include dry mouth and constipation. Rare but serious adverse events include increased intraocular pressure and acute glaucoma (0.06\%) [11].

The population of Korea is rapidly aging. As a result, the number of patients with irritable bladder and taking antimuscarinic drugs is expected to increase. This study examined patients with various underlying diseases to confirm the infrequency of uncommon adverse events, such as acute glaucoma. The effects of solifenacin fumarate on irritable bladder symptoms were also investigated.

\section{MATERIALS AND METHODS}

\section{Study Patients}

From January 1, 2018 to December 31, 2018, 163 patients who received solifenacin fumarate from the urology outpatient department of the study institution were enrolled in this study. The medical records of patients who completed questionnaires on the OABSS and PPBC score before and after administration were analyzed.

This study was a retrospective multi-institutional observation study that evaluated the medical records. This study was approved by the Institutional Review Board of the Chung-Ang University Hospital (No. 1907-007-16272). Consent from the patients was impossible because the patient did not visit the institution for this study. Furthermore, there 
was no reason to estimate the rejection of consent, and there was no risk to the patients in this study. For this reason, consent from the patients was not required.

\section{Specific Procedures}

Patients who met the selection/exclusion criteria were admitted to the urology outpatient clinic and administered solifenacin fumarate $5 \mathrm{mg}$. They were given a questionnaire on the self-reported bladder symptoms (using the PPBC). They self-evaluated their OABSSs before and after solifenacin fumarate $5 \mathrm{mg}$ intake. The patients' medical records and questionnaire responses were analyzed to evaluate the effectiveness and safety.

The exclusion criteria were as follows: patients who were pregnant or planning to become pregnant (within 12 months) and patients with a history of hypersensitivity to solifenacin fumarate; urinary obstructive symptoms; glaucoma; myasthenia gravis; pyloric, duodenal, or other intestinal obstruction or paralytic intestinal obstruction; gastric atrophy or intestinal dilatation; addictive giant colon; severe heart disease; and

Table 1. General characteristics of the subjects

\begin{tabular}{lc}
\hline \multicolumn{1}{c}{ Characteristic } & Value $(\mathrm{n}=163)$ \\
\hline Age $(\mathrm{y})$ & $58.6 \pm 17.1$ \\
Male & $68(41.7)$ \\
Height $(\mathrm{cm})$ & $161.4 \pm 8.7$ \\
Weight $(\mathrm{kg})$ & $61.3 \pm 10.5$ \\
Body mass index $\left(\mathrm{kg} / \mathrm{m}^{2}\right)$ & $23.4 \pm 3.0$ \\
Diabetes mellitus & $21(12.8)$ \\
Intake of $\alpha$-blocker & $12(7.3)$ \\
Onset duration $(\mathrm{mo})$ & $26.4 \pm 29.1$ \\
\hline
\end{tabular}

Values are presented as mean \pm standard deviation or number (\%).

A

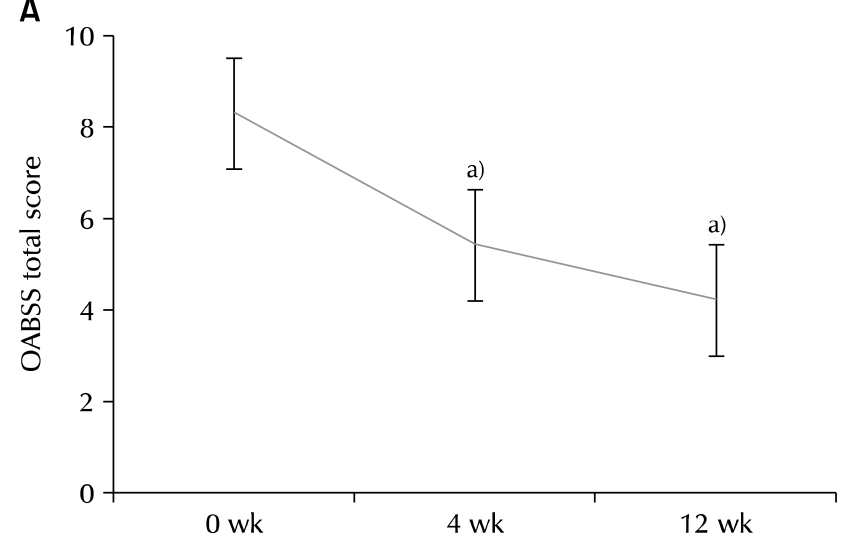

severe liver failure. The patients' age, sex, height, weight, body mass index (BMI), DM and hypertension status, alpha-blocker use, and onset duration (months) were evaluated.

The investigation was conducted three times. The observation period for each research subject was $12 \pm 4$ weeks. Investigation 1 was defined as the baseline (w0), with solifenacin fumarate taken on the first day of the study. Investigation 2 was defined as $4 \pm 1$ week (w4) from the start date, and Investigation 3 was defined as $12 \pm 2$ weeks (w12) from the start date.

There were three types of survey items: the OABSS, PPBC score, and abnormal cases. The OABSS captured the number of times that a person passed urine each day (Q1) and each night (Q2), urgency (Q3), and urinary incontinence due to urgency (Q4). A higher number indicated worse symptoms. The PPBC score, with outcomes ranging from 1 to 6 , indicates the bladder condition in terms of the degree of discomfort in everyday life. Like the OABSS, a higher number indicated poorer symptoms. Some questionnaire items aimed to capture adverse events in terms of type, date of onset, date of resolution, severity, outcome, causality, related action, and whether there was corrective treatment. The severity of drug-related adverse events was classified using the National Cancer Institute's common terminology criteria.

\section{Statistical Analysis}

IBM SPSS Statistics version 21.0 (IBM Co., Armonk, NY, USA) was used for all statistical analysis. The statistical significance was verified using paired t-tests comparing the findings among the designated time points. The adverse

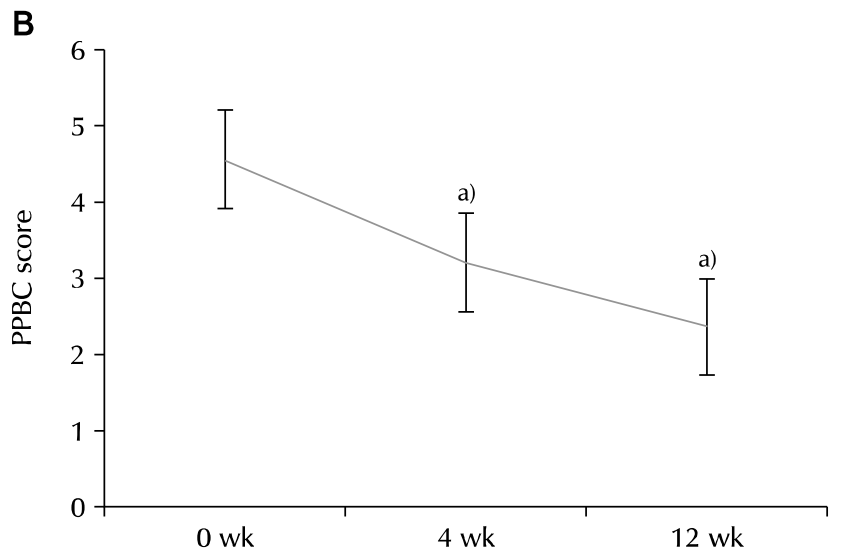

Fig. 1. (A) Variation of the Overactive Bladder Symptom Score (OABSS) total score. (B) Variation of Patient Perception of Bladder Condition (PPBC) score. ${ }^{\text {a) }}$ Statistically significant. 
events were classified according to the timing of the adverse events and their severity.

\section{RESULTS}

This study surveyed 163,140 , and 117 patients at the baseline w0, w4, and w12, respectively. The mean age was $58.6 \pm 17.1$ years, and 68 patients $(41.7 \%)$ were male. The mean height, weight, and BMI were $161.4 \pm 8.7 \mathrm{~cm}, 61.3 \pm 10.5$ $\mathrm{kg}$, and $23.4 \pm 3.0 \mathrm{~kg} / \mathrm{m}^{2}$, respectively. Twenty-one patients (12.8\%) were diagnosed with DM. Twelve patients (7.3\%) were on alpha-blocker treatment, and the mean duration from the onset of symptoms to the start of administration was $26.4 \pm 29.1$ months (Table 1).

The more solifenacin fumarate a patient took, the more all of the outcome measures decreased. The mean OABSS score (total) decreased from $8.45 \pm 2.38(\mathrm{p}=0.199)$ at w0, to $5.41 \pm 2.69(\mathrm{p}=0.255)$ at $\mathrm{w} 4$, and $4.21 \pm 2.61(\mathrm{p}=0.240)$ at $\mathrm{w} 12$ (Fig. 1A). In the mean of the bladder self-assessment score showed a decreasing trend: $4.55 \pm 0.81(\mathrm{p}=0.068)$ at $\mathrm{w} 0$, $3.19 \pm 0.78(\mathrm{p}=0.087)$ at $\mathrm{w} 4,2.34 \pm 0.87(\mathrm{p}=0.080)$ at $\mathrm{w} 12$ (Fig. 1B). Looking at each question, the mean OABSS scores for Q1 at w0, w4, and w12 were 1.19 \pm 0.61 ( $\mathrm{p}=0.051), 0.86 \pm 0.62$ $(\mathrm{p}=0.052)$, and $0.76 \pm 0.57(\mathrm{p}=0.053)$, respectively (Fig. 2A). The mean OABSS scores for Q2 at w0, w4, and w12 were

Table 2. Side effects after each intervention

\begin{tabular}{ccc}
\hline Side effect & 4 weeks $(\mathrm{n}=140)$ & 12 weeks $(\mathrm{n}=117)$ \\
\hline Dry mouth & $3(2.1)$ & $5(4.2)$ \\
Grade 1 & $3(2.1)$ & $1(0.8)$ \\
Grade 2 & $0(0)$ & $0(0)$ \\
Grade 3 & & \\
Constipation & $1(0.7)$ & $3(1.6)$ \\
Grade 1 & $3(2.1)$ & $0(0)$ \\
Grade 2 & $0(0)$ & \\
Grade 3 & & $1(0)$ \\
Hydroblepharon & $0(0)$ & $0(0)$ \\
Mild & $0(0)$ & $0(0)$ \\
Moderate & $0(0)$ & \\
Severe & &
\end{tabular}

Values are presented as number (\%).
A



C

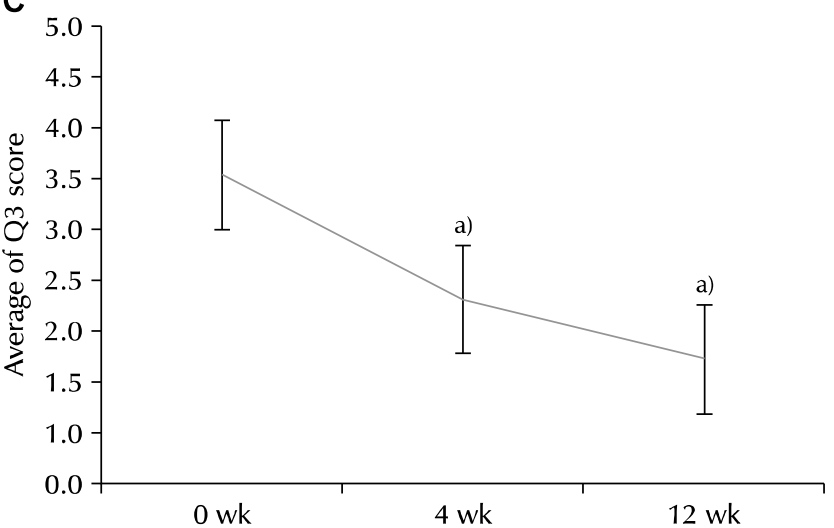

B

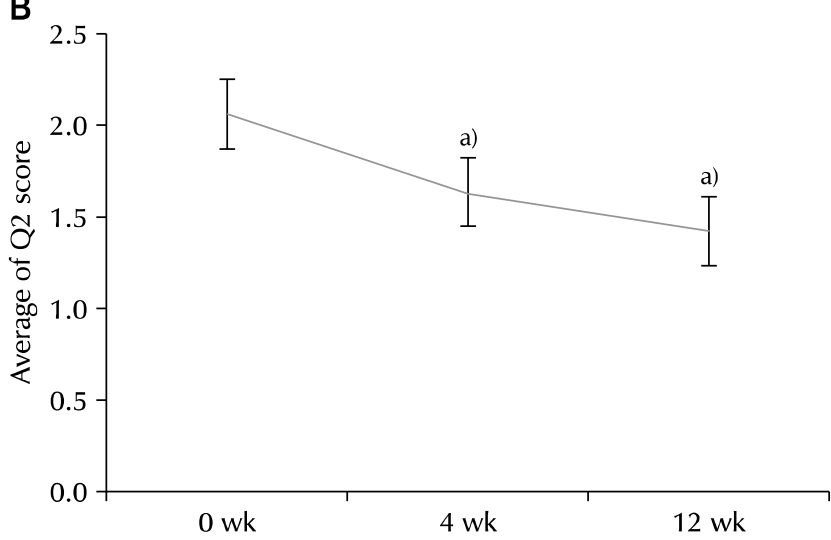

D

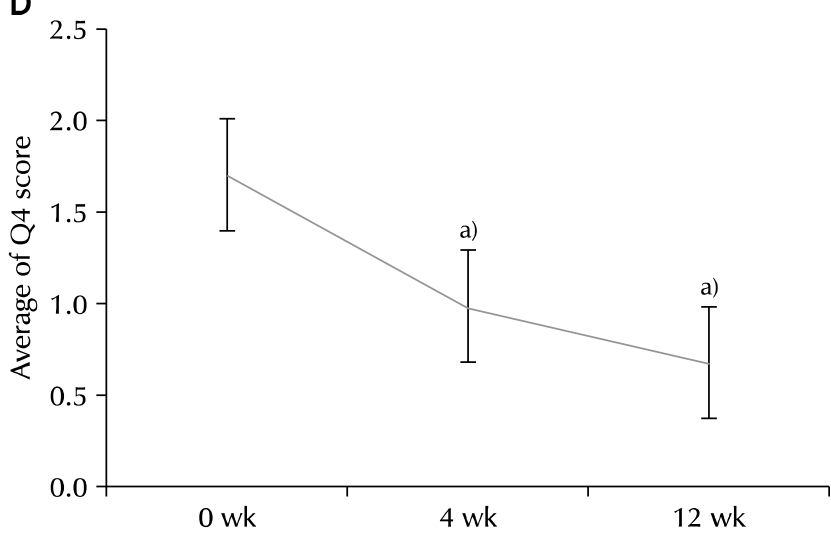

Fig. 2. Changes in the Overactive Bladder Symptom Score (OABSS) over time. (A) OABSS score (Q1). (B) OABSS score (Q2). (C) OABSS score (Q3). (D) OABSS score (Q4). Q1: frequency, Q2: nocturia, Q3: urgency, Q4: incontinence. ${ }^{\text {a) }}$ Statistically significant. 
$2.19 \pm 0.93(\mathrm{p}=0.078), 1.63 \pm 0.95(\mathrm{p}=0.080)$, and $1.42 \pm 0.95$ $(\mathrm{p}=0.087)$, respectively (Fig. $2 \mathrm{~B})$. The mean OABSS scores for Q3 at w0, w4, and w12 were 3.34 \pm 1.05 ( $\mathrm{p}=0.088$ ), $2.00 \pm 1.28(\mathrm{p}=0.108)$, and $1.35 \pm 1.06(\mathrm{p}=0.098)$, respectively (Fig. 2C). The mean OABSS scores for Q4 at w0, w4, and w12 were $1.74 \pm 1.50(\mathrm{p}=0.126), 0.98 \pm 1.12(\mathrm{p}=0.094)$, and $0.66 \pm 0.92$ ( $\mathrm{p}=0.085$ ), respectively (Fig. 2D).

Some adverse events were encountered (Table 2). Dry mouth was most common, followed by constipation and eyelid edema. None of the patients in the study had any other side effects. Grade 1 and 2 dry mouth occurred in three patients each $(2.1 \%)$ at $\mathrm{w} 4$ and in five patients (4.2\%) and one person (0.8\%), respectively, at w12. The drug dose was reduced for one patient in response to a dry mouth. One patient permanently stopped taking solifenacin fumarate. No patients received medical treatment for dry mouth. Grade 1 and 2 constipation occurred in one person (0.7\%) and three patients (2.1\%), respectively, at $\mathrm{w} 4$, and in two patients (1.7\%) and three patients (2.5\%), respectively, at w12. Regarding constipation, four, one, five, and two patients were maintained on the drug, had their dosage reduced, restarted the drug after initially stopping, and permanently stopped taking solifenacin fumarate, respectively. The corrective treatments for constipation included one patient taking lactobacillus, two patients taking laxatives, and one patient taking medical treatment. One person had eyelid edema after taking solifenacin fumarate. The drug was discontinued, but corrective treatment was not performed. The above side effects took approximately two to four weeks from when the symptoms occurred to the time they disappeared.

\section{DISCUSSION}

The pathogenetic mechanism of overactive bladder is unclear, but it is believed to arise from hyperactivity of the sensory bladder function and detrusor muscle. Bladder fullness was thought to be sensed at the ends of the sensory nerves distributed on the bladder wall, but it was recently discovered that the bladder mucosa and submucosal interstitial cells play important roles in the bladder sensory mechanisms [8]. The sensory innervation of the bladder is distributed throughout the detrusor muscle, bladder mucosa, submucosal tissue, and submucosal interstitial cells. As the bladder elongates, the bladder mucosa secretes more acetylcholine, adenosine triphosphate, and prostaglandin.
The receptors for these ligands are distributed in the submucosal interstitial cells and sensory nerves. The activity of the sensory nerves is regulated according to the concentration of these substances. Aging, irritable bladder, outlet obstruction, and central nerve damage are associated with the increased secretion of these compounds from the bladder mucosa, and they stimulate the sensory nerve endings under the bladder mucosa, leading to urgency. Detrusor muscle hyperactivity arises from neurogenic and myogenic factors. Cerebral and spinal processes regulate detrusor muscle contractions. The detrusor muscle causes denervation and changes in intercellular binding associated with aging, neurological disease, bladder outlet obstruction, and ischemia. Denervation causes hyperreactivity of the detrusor muscle to acetylcholine, causing autonomically mediated contraction. Increased intercellular binding delivers the autonomic contraction of the detrusor muscle to the surrounding detrusor muscle, resulting in the contraction of the detrusor muscle over a wide area.

Solifenacin fumarate is a product in which the salt component of solifenacin succinate is changed. In terms of the median lethal dose $\left(\mathrm{LD}_{50}\right)$, fumarate is four times safer than succinate. Solifenacin fumarate intake improves the symptoms of bladder hypersensitivity, which were confirmed by the PPBC and OBASS outcomes. Side effects, including dry mouth, constipation, and eyelid edema, affected less than 10 ( $\mathrm{n}=16)$ of the study patients, and no grade 3 adverse events were reported, supporting the safety of the drug.

Dry mouth and constipation have been reported as the most common side effects of solifenacin and tolterodine in review papers and randomized controlled trials comparing solifenacin and tolterodine [12]. In the case of dry mouth as a side effect, one study found no significant difference in the standard mean differences (SMDs) between the designated time points: $0.66(\mathrm{p}=0.060)$ at $\mathrm{w} 4$ and $0.84(\mathrm{p}=0.350)$ at w12. For constipation, the SMDs were $0.63(\mathrm{p}=0.180)$ at $\mathrm{w} 4$ and $2.96(\mathrm{p}=0.006)$ at $\mathrm{w} 12$. Constipation occurred less frequently in association with tolterodine than with solifenacin. In the present study, constipation occurred in six out of 151 (3.97\%) patients at w4 and six out of 118 $(5.08 \%)$ at $w 12$.

A meta-analysis conducted to analyze the efficacy and safety of solifenacin $(5 \mathrm{mg})$ and mirabegron $(50 \mathrm{mg})$ monotherapy for 12 weeks for overactive bladder found that solifenacin and mirabegron had similar effects in terms of 
daily incontinence-associated leakage episodes, daily micturition frequency, daily urgency episodes, and urine volume during a single void. In terms of safety, the incidence of drug-related treatment-emergent adverse events was significantly higher in the solifenacin group, but there was no significant difference in the overall side effects between the groups. The mean post-voiding residual volume was significantly higher in the solifenacin group. Similarly, dry mouth was higher in the solifenacin group, but the two groups did not differ significantly in terms of odd ratio for visual field abnormalities and constipation [13].

A 12-month double-blind phase 3 study comparing solifenacin and mirabegron combination therapy with monotherapy regimens of each drug found that solifenacin succinate $(5 \mathrm{mg})$ and mirabegron $(50 \mathrm{mg})$ combination therapy had a significantly better effect on urinary incontinence and urination than either monotherapy. On the other hand, the incidence of side effects was higher in the combination therapy group (49\%) than the mirabegron group (41\%) and solifenacin group (44\%). Dry mouth was the most common side effect associated with combination therapy and monotherapy. Other side effects included rhinitis (combination group, 3.6\%; solifenacin group, 5.0\%; mirabegron group, 5.2\%), urinary tract infection (combination group, 3.4\%; solifenacin group, 4.0\%; mirabegron group, 3.6\%), and constipation (combination group, 3.3\%; solifenacin group, 2.3\%; mirabegron group, 1.3\%) [14]. Mirabegron can be added to the regimen if symptoms do not improve after solifenacin administration, but this may increase the likelihood of adverse events compared to solifenacin monotherapy.

Currently, there are many antimuscarinic agents as $\mathrm{OAB}$ drugs. Among them, the effectiveness and tolerability of propiverine and solifenacin were compared [15]. That study was a prospective non-randomized crossover study of propiverine $20 \mathrm{mg}$ and solifenacin $5 \mathrm{mg}$ for female $\mathrm{OAB}$ patients. The patients were administered propiverine or solifenacin for eight weeks. The propiverine group was changed to solifenacin (group P-S), and the solifenacin group was changed to propiverine (group S-P) for another eight weeks. The OABSS was used to evaluate the OAB symptoms. In both groups, the OABSS total score and each item score were improved. In particular, in group P-S, the urgency score at 16 weeks was improved significantly compared to that at eight weeks. Therefore, solifenacin had better efficacy than propiverine.

Muscarinic receptors play an important role in cognitive functions, such as memory and learning [16]. Because the muscarinic receptor is in the central nervous system, cognitive impairment can occur as a side effect of anticholinergic agents, particularly in older people. Solifenacin was reported to be good at central nervous system penetration in an in vivo study, but some clinical studies suggested that it is not significantly related to cognitive function [17]. Rather, one study suggested that patients taking donepezil for Alzheimer's disease can use it safely without deteriorating their cognitive function [18].

This study had some limitations. Because the study was not conducted with a control group, it was impossible to compare the superiority or inferiority of solifenacin with those of other drugs. Furthermore, because the follow-up was limited to 12 weeks, the longer-term effects were not investigated. Hence, future research is warranted in this regard. Nevertheless, the number of study subjects was sufficient, and solifenacin showed significant efficacy without causing serious side effects. Therefore, a welldesigned study will be needed in the future.

\section{CONCLUSIONS}

Solifenacin fumarate is more stable as an active drug than solifenacin succinate. Increased solifenacin fumarate intake was associated with better self-assessed PPBC and OABSS outcomes. Moreover, solifenacin was associated with a low incidence of mild adverse events. The study findings support the efficacy and safety of solifenacin as a treatment for overactive bladder.

\section{CONFLICT OF INTEREST}

No potential conflict of interest relevant to this article was reported.

\section{ACKNOWLEDGMENTS}

This study was sponsored by Ahngook Pharmaceutical Co. Ltd, Seoul, Republic of Korea.

Qualified investigators at the Department of Urology of Chung-Ang University Hospital performed the clinical trials. 


\section{AUTHOR CONTRIBUTIONS}

J.H.S. performed the statistical analysis and wrote the manuscript. S.Y.C. and J.H.G. review the manuscript. Y.J.K., W.H.C., D.H.K., K.H.K., Y.W.P., J.M.U., I.S.L., K.K.S., K.S.C., Y.J.L., M.K.L., and T.H.K. participated in the study design and coordination and helped to draft the manuscript. All authors read and approved the final manuscript.

\section{ORCID}

Jae Hun Shim, https://orcid.org/0000-0003-2307-4136 Se Young Choi, https://orcid.org/0000-0002-4615-0966 Joon Hee Gook, https://orcid.org/0000-0003-3483-5893 Yong-June Kim, https://orcid.org/0000-0001-7638-7174 Woo Heon Cha, https://orcid.org/0000-0002-9119-5178 Dae Hee Kim, https://orcid.org/0000-0001-8528-6375 Kyeong Hee Kim, https://orcid.org/0000-0002-3602-7614 Young Woong Park, https://orcid.org/0000-0002-6246-9133 Jin Mo Um, https://orcid.org/0000-0001-8191-6237 Il Sung Lim, https://orcid.org/0000-0001-7715-9667 Kyung Keun Seo, https://orcid.org/0000-0002-7839-5810 Kyu Seon Cho, https://orcid.org/0000-0002-1368-0186 Young Jae Lee, https://orcid.org/0000-0002-4032-9121 Mi-Kyung Lee, https://orcid.org/0000-0003-1824-476X Tae-Hyoung Kim, https://orcid.org/0000-0002-0257-3449

\section{REFERENCES}

1. Austin PF, Bauer SB, Bower W, Chase J, Franco I, Hoebeke P, et al. The standardization of terminology of lower urinary tract function in children and adolescents: update report from the standardization committee of the International Children's Continence Society. Neurourol Urodyn 2016;35:471-81.

2. Milsom I, Abrams P, Cardozo L, Roberts RG, Thüroff J, Wein AJ. How widespread are the symptoms of an overactive bladder and how are they managed? A population-based prevalence study. BJU Int 2001;87:760-6.

3. Lee YS, Lee KS, Jung JH, Han DH, Oh SJ, Seo JT, et al. Prevalence of overactive bladder, urinary incontinence, and lower urinary tract symptoms: results of Korean EPIC study. World J Urol 2011;29:185-90.

4. Irwin DE, Milsom I, Hunskaar S, Reilly K, Kopp Z, Herschorn S, et al. Population-based survey of urinary incontinence, overactive bladder, and other lower urinary tract symptoms in five countries: results of the EPIC study. Eur Urol 2006; 50:1306-14; discussion 1314-5.
5. Melotti IGR, Juliato CRT, Tanaka M, Riccetto CLZ. Severe depression and anxiety in women with overactive bladder. Neurourol Urodyn 2018;37:223-8.

6. Kinsey D, Pretorius S, Glover L, Alexander T. The psychological impact of overactive bladder: a systematic review. J Health Psychol 2016;21:69-81.

7. Szabo SM, Gooch KL, Walker DR, Johnston KM, Wagg AS. The association between overactive bladder and falls and fractures: a systematic review. Adv Ther 2018;35:1831-41.

8. Matza LS, Thompson CL, Krasnow J, Brewster-Jordan J, Zyczynski T, Coyne KS. Test-retest reliability of four questionnaires for patients with overactive bladder: the overactive bladder questionnaire (OAB-q), patient perception of bladder condition (PPBC), urgency questionnaire (UQ), and the primary OAB symptom questionnaire (POSQ). Neurourol Urodyn 2005;24:215-25.

9. Matsukawa Y, Majima T, Takai S, Funahashi Y, Kato M, Gotoh M. What are factors contributing to improvement of overactive bladder symptoms after alpha-1 blocker treatment in patients with both storage and voiding symptoms? Urol Int 2019; 103:439-43.

10. Chapple CR, Araño P, Bosch JL, De Ridder D, Kramer AE, Ridder AM. Solifenacin appears effective and well tolerated in patients with symptomatic idiopathic detrusor overactivity in a placeboand tolterodine-controlled phase 2 dose-finding study. BJU Int 2004;93:71-7.

11. Sekeroglu MA, Hekimoglu E, Petricli IS, Tasci Y, Dolen I, Arslan $U$. The effect of oral solifenacin succinate treatment on intraocular pressure: glaucoma paradox during overactive bladder treatment. Int Urogynecol J 2014;25:1479-82.

12. Chapple CR, Martinez-Garcia R, Selvaggi L, Toozs-Hobson P, Warnack W, Drogendijk T, et al.; STAR study group. A comparison of the efficacy and tolerability of solifenacin succinate and extended release tolterodine at treating overactive bladder syndrome: results of the STAR trial. Eur Urol 2005;48:464-70.

13. Wang J, Zhou Z, Cui Y, Li Y, Yuan H, Gao Z, et al. Meta-analysis of the efficacy and safety of mirabegron and solifenacin monotherapy for overactive bladder. Neurourol Urodyn 2019;38:22-30.

14. Gratzke C, van Maanen R, Chapple C, Abrams P, Herschorn S, Robinson $\mathrm{D}$, et al. Long-term safety and efficacy of mirabegron and solifenacin in combination compared with monotherapy in patients with overactive bladder: a randomised, multicentre phase 3 study (SYNERGY II). Eur Urol 2018;74:501-9.

15. Wada N, Watanabe M, Kita M, Osanai H, Yamaguchi S, Numata A, et al.; OAB Study Group in Northern Hokkaido. Efficacy and safety of propiverine and solifenacin for the treatment of female patients with overactive bladder: a crossover study. Low Urin Tract Symptoms 2011;3:36-42.

16. Kim YJ, Tae BS, Bae JH. Cognitive function and urologic medications for lower urinary tract symptoms. Int Neurourol J 
2020;24:231-40.

17. Burger M, Betz D, Hampel C, Vogel M. Efficacy and tolerability of solifenacin in men with overactive bladder: results of an observational study. World J Urol 2014;32:1041-7.
18. Hampel C, Betz D, Burger M, Nowak C, Vogel M. Solifenacin in the elderly: results of an observational study measuring efficacy, tolerability and cognitive effects. Urol Int 2017; 98:350-7. 